\title{
Wechselwirkungen zwischen Mindest- und Tariflöhnen
}

\author{
Länderunterschiede bei der Lohnungleichheit lassen sich mit verschiedenen Institutionen \\ der Lohnfindung erklären. In diesem Artikel wird analysiert, welchen Beitrag Mindest- \\ löhne und Tarifverträge zur Verringerung von Lohnungleichheit leisten und welche $\mathrm{Zu}$ - \\ sammenhänge und Wechselwirkungen zwischen beiden bestehen. Das Zusammenwirken \\ von Mindestlöhnen und Tarifverträgen in der Architektur nationaler Lohnsysteme wird \\ am Beispiel ausgewählter Länder untersucht und auf dieser Basis eine Länder-Typologie \\ entwickelt, die hilft, unterschiedliche Wechselwirkungen von Mindest- und Tariflöhnen \\ zu verstehen.
}

GERHARD BOSCH, CLAUDIA WEINKOPF

\section{Einleitung}

Wie lassen sich die deutlichen Länderunterschiede im Ausmaß und in der Entwicklung der Lohnungleichheit erklären? Universelle Erklärungsansätze wie Globalisierung oder die These der skill-biased technologischen Entwicklung, also die technologisch bedingte Abnahme der Nachfrage nach einfacher Arbeit, sind aus verschiedenen Gründen wenig hilfreich. So gibt es keine Belege dafür, dass die Globalisierung, gemessen an der Intensität der Handelsverflechtungen, sowie das Tempo des technologischen Fortschritts in Ländern mit wenig Niedriglohnbeschäftigung deutlich geringer ausgeprägt sind (Blau/Kahn 1994). Zudem ist schlecht bezahlte Arbeit nicht notwendigerweise gering qualifiziert. So haben in Deutschland rund 80 \% der Niedriglohnbeschäftigten eine Berufsausbildung oder sogar einen akademischen Abschluss (Kalina/Weinkopf 2012). Das Ausmaß von Niedriglöhnen korreliert weiterhin auch nicht mit dem Bruttosozialprodukt pro Kopf oder dessen Wachstumsrate, der Arbeitsproduktivität pro Stunde oder wichtigen demografischen Faktoren wie etwa der Frauenerwerbsbeteiligung. Ebenso wenig lassen sich Länderunterschiede durch unterschiedliche Branchenstrukturen oder den Anteil der Arbeitskosten an der gesamten Wertschöpfung erklären (z. B. Salverda/Mayhew 2009).

Signifikant sind hingegen die Zusammenhänge zwischen Lohnungleichheit und Institutionen der Lohnfindung.
Die Wirkungen der Globalisierung, des technischen Fortschritts, unterschiedlicher Wirtschaftsstrukturen sowie externer Schocks, wie etwa die Deregulierung von Gütermärkten oder die Privatisierung öffentlicher Dienstleistungen, werden durch nationale Lohnsysteme "gefiltert", was zu national sehr unterschiedlichen Wirkungen führt. Die Zunahme geringer Löhne kann fast völlig mit der Schwächung dieser Institutionen erklärt werden (Sniekers 2011). Es ist auch bekannt, dass Mindestlöhne vor allem die Lohnverteilung im unteren Bereich beeinflussen, während Tariflöhne eher Wirkungen im mittleren Bereich entfalten.

Während sich zahlreiche Untersuchungen zu den Beschäftigungswirkungen von Mindestlöhnen finden, sind die Interaktionen zwischen Mindestlöhnen und Tarifverträgen und ihre gemeinsamen Auswirkungen auf die Einkommensverteilung bislang hingegen kaum erforscht. Erst seit einigen Jahren werden die Wirkungen von Mindestlöhnen auf die darüber liegenden Löhne, die sogenannten ripple-Effekte, empirisch untersucht. Wicks-Lim (2008) fand heraus, dass Erhöhungen des US-amerikanischen Mindestlohnes Auswirkungen bis zum vierzigsten Perzentil in der Lohnverteilung haben und dass die Auswirkungen besonders stark in Branchen sind, in denen sich die Löhne knapp über dem Mindestlohn konzentrieren. Stewart (2010) konnte hingegen im Vereinigten Königreich keine ripple-Effekte von Mindestlöhnen feststellen. Seine quantitativen Ergebnisse werden durch Fallstudien in typischen Niedriglohn-Branchen untermauert (Grimshaw 2010). Diese zeigen, 
dass Lohndifferenzen nicht wiederhergestellt worden und sogar negative ripple-Effekte aufgetreten sind, da der Abstand zwischen dem gesetzlichen Mindestlohn und den darüber liegenden Löhnen abgenommen hat. Die Gründe hierfür sind in der Schwächung des britischen Tarifsystems und der Aufstockung geringer Löhne durch staatliche Subventionen (in-work benefits) zu sehen, die das Interesse der Beschäftigten, höhere Löhne auszuhandeln, verringern. Koubi/Lhommeau (2007) stellten für Frankreich fest, dass eine Erhöhung des Mindestlohns (SMIC) zu Erhöhungen der Löhne bis zum zweifachen des SMIC mit abnehmender Quote führte. Die neuere deutsche Mindestlohnforschung zu den Auswirkungen von Branchenmindestlöhnen hat gezeigt, dass in Branchen mit hohem Fachkräfteanteil Erhöhungen der Mindestlöhne die Lohnkurve in Westdeutschland nach oben verschoben haben mit beträchtlichen ripple-Effekten bis deutlich über den Medianlohn der Branche hinaus, während in Ostdeutschland die Lohnstruktur komprimiert wurde mit zum Teil deutlichen Verlusten bei den höheren Löhnen (Bosch/Weinkopf 2012a).

In Lohntheorien wird auf die Bedeutung von sogenannten Ecklöhnen (key rates) verwiesen, an denen sich die Löhne anderer Beschäftigter orientieren (Dunlop 1957). Die Löhne der Beschäftigten mit besserer Qualifikation, längerer Betriebszugehörigkeit oder höherer Stellung in der Hierarchie steigen, wenn sich diese Ecklöhne innerhalb von Lohnkonturen (wage contours) verändern. Gemeint sind einigermaßen stabile Lohnstrukturen in Branchen, Berufsgruppen oder Regionen (Dunlop 1957, S. 131). Dunlop konnte selbst für die USA im Jahr 1957 noch davon ausgehen, dass „Tarifverhandlungen als der Normalfall angesehen werden müssen“ (ebd. S. 125, Übersetzung G.B./C.W.), sodass er die Lohnkonturen damals noch mit den Gehaltsrahmentarifverträgen gleichsetzte, in denen eine Erhöhung der Ecklöhne das gesamte Tarifgitter in festen Proportionen nach oben verschob. Mit der Schwächung der Gewerkschaften sowie der Dezentralisierung und Individualisierung der Lohnfindung sind die Lohnkonturen jedoch in vielen Ländern informeller und vor allem instabiler geworden, sodass heute in den OECD-Ländern die Spannbreite von positiven bis hin zu negativen ripple-Effekten reicht und sich diese Effekte auch im Zeitablauf ändern können.

Wissenschaftliche Analysen aus Ländern mit geringer Tarifbindung und hohen betrieblichen Entscheidungsspielräumen über Lohnstrukturen begründen positive rippleEffekte vor allem mit dem Interesse der Unternehmen, ihre interne Lohndifferenzierung zu bewahren. Um die Arbeitsmoral, Motivation und Produktivität der Beschäftigten zu erhalten, müssten auch die Löhne der besser Qualifizierten angehoben werden (Wicks-Lim 2008, S. 199; Stewart 2010, S. 2). Dies hat im Vereinigten Königreich aber offenbar viele Unternehmen nicht davon abgehalten, die Kosten einer Erhöhung des Mindestlohns durch eine Absenkung der darüber liegenden Löhne zu finanzieren. Da der gesetzliche Mindestlohn in Frankreich in vielen Branchen nahe an den untersten Tariflöhnen liegt, wird bei einer Mindestlohner- höhung in diesen Branchen das Tarifgefüge insgesamt angehoben. Daher verwundern die hohen ripple-Effekte in Frankreich nicht. Insgesamt sprechen die beschriebenen Befunde dafür, dass die Interaktionen zwischen Mindestlöhnen und Tarifverträgen ganz unterschiedlich sind und von der jeweiligen Architektur der Institutionen der Lohnfindung und der Stärke und dem Handeln zentraler Akteure abhängen (Grimshaw/Bosch 2013).

In diesem Beitrag gehen wir der Frage nach, welchen Beitrag Mindestlöhne und Tarifverträge zur Verringerung von Lohnungleichheit leisten und welche Zusammenhänge und Wechselwirkungen zwischen beiden bestehen. In Abschnitt 2 analysieren wir zunächst jeweils getrennt den Einfluss von gesetzlichen Mindestlöhnen einerseits und Tarifbindung andererseits auf die Lohnungleichheit. In Abschnitt 3 untersuchen wir das Zusammenwirken von Mindestlöhnen und Tarifverträgen in der Architektur nationaler Lohnsysteme am Beispiel ausgewählter Länder und entwickeln auf dieser Basis eine Typologie, die hilft, positive und negative ripple-Effekte von Mindestlöhnen zu verstehen. Am Beispiel Deutschlands zeigen wir, wie vielfältig diese Interaktionen innerhalb von Ländern sein können (4). Abschließend (5) werden die zentralen Ergebnisse zusammengefasst und einige Schlussfolgerungen für den weiteren Forschungsbedarf gezogen.

\section{Die Auswirkungen von Mindest- löhnen und Tarifbindung auf die Lohnungleichheit}

Durch gesetzliche Mindestlöhne werden Lohnuntergrenzen gesetzt, die nicht unterschritten werden dürfen. Die unmittelbaren Wirkungen von Mindestlöhnen sind damit in der Kompression der Löhne am unteren Ende der Lohnverteilung zu sehen. Mindestlöhne können den Anteil der Niedriglohnbeschäftigten nur verringern, wenn ihr Niveau nahe an der Niedriglohnschwelle (zwei Drittel des Medianlohnes) liegt. Da gesetzliche Mindestlöhne sich meist an der wirtschaftlichen Leistungsfähigkeit der schwächeren Branchen orientieren, werden sie i. d. R. sehr vorsichtig angehoben und liegen in der Praxis überwiegend deutlich unterhalb der Niedriglohnschwelle. Mindestlöhne können darüber hinaus die interpersonelle Einkommensverteilung beeinflussen, wenn bestimmte Personengruppen überproportional von sehr niedrigen Löhnen betroffen sind wie etwa Frauen, gering Qualifizierte oder Beschäftigte mit Migrationshintergrund und damit von der Einführung oder Anhebung einer Lohnuntergrenze besonders profitieren (Grimshaw/Rubery 2013).

Das Niveau von Mindestlöhnen wird durch den sogenannten Kaitz-Index gemessen. Dieser drückt den relativen Anteil des Mindestlohns zum Medianlohn aus. Abbildung 1 
zeigt den Zusammenhang zwischen dem Kaitz-Index der Mindestlöhne in unterschiedlichen Ländern und dem jeweiligen Anteil der Niedriglohnbeschäftigung für das Jahr 2010. Der Zusammenhang zwischen der Höhe der Mindestlöhne und dem Niedriglohnanteil ist mit einer Korrelation von $-0,34$ nur sehr schwach. ${ }^{1} \mathrm{Zu}$ ähnlichen Ergebnissen kommen auch Salverda/Mayhew (2009, S. 147) und die Europäische Kommission (European Commission 2009, Kapitel 3). Nur in Frankreich liegt der Mindestlohn so nah an der Niedriglohnschwelle, dass er einen eigenständigen Beitrag zur Begrenzung des Niedriglohnanteils leistet. In den anderen EU-Ländern wird durch die Mindestlöhne der Niedriglohnanteil nicht verringert, aber das Ausfransen der niedrigsten Löhne nach unten begrenzt.

Stärker ist der Einfluss von Mindestlöhnen auf die Lohnverteilung zwischen Männern und Frauen. Hier liegt die Korrelation zwischen der Höhe des Kaitz-Index und dem Niedriglohnanteil von Frauen bei immerhin -0,52 (Abbildung 2). In Ländern mit vergleichsweise geringen Mindestlöhnen (gemessen am Medianlohn von Vollzeitbeschäftigten) ist die Lohnungleichheit zwischen Männern und Frauen besonders groß wie etwa in Litauen (LT). Die positiven Auswirkungen von Mindestlöhnen auf die Lohnunterschiede zwischen Männern und Frauen waren auch Gegenstand der Evaluation verschiedener nationaler Mindestlöhne. So verringerte sich in Großbritannien beispielsweise der Lohnunterschied zwischen Männern und Frauen nach der Einführung des nationalen gesetzlichen Mindestlohns im Jahre 1999. Verdienten vollzeitbeschäftig-

ABB. 1

Relative Höhe des Mindestlohns (Kaitz-Index) und Niedriglohnanteil der Beschäftigten 2010

Angaben in Prozent

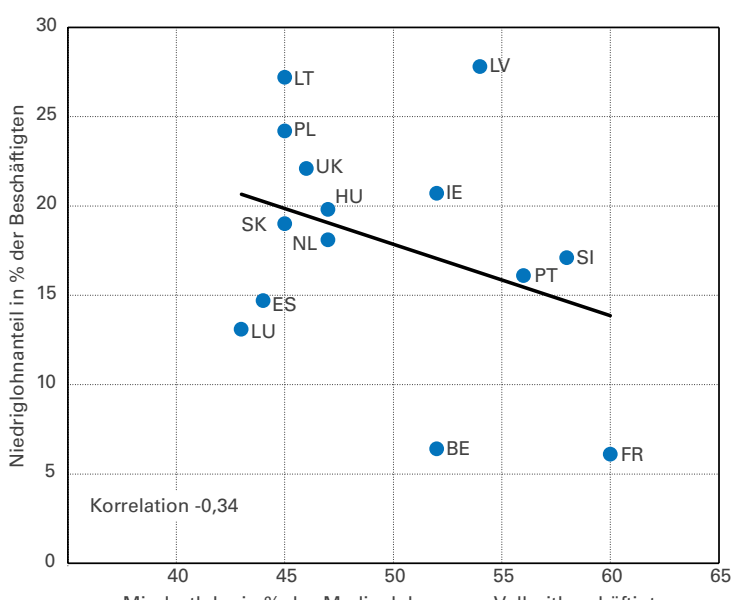

Mindestlohn in \% des Medianlohns von Vollzeitbeschäftigten

$\mathrm{BE}=$ Belgien, $\mathrm{ES}=\mathrm{Spanien}, \mathrm{FR}=$ Frankreich, $\mathrm{HU}=$ Ungarn, IE=Irland, LT=Litauen, $\mathrm{LU}=\mathrm{Luxemburg}, \mathrm{LV}=\mathrm{Lettl}$ and, NL=Niederlande, PL=Polen, PT=Portugal, SI=Slowenien, SK=Slowakei, UK=Großbritannien

Quelle: Schulten 2012a (Kaitz-Index); Bezzina 2012 (Niedriglohnanteil); Berechnungen der Autoren.

$\mathrm{BE}=$ Belgien, $\mathrm{ES}=\mathrm{Spanien}, \mathrm{FR}=$ Frankreich, $\mathrm{HU}=$ Ungarn, IE=Irland, LT=Litauen $\mathrm{LU}=$ Luxemburg, LV=Lettland, NL=Niederlande, PL=Polen, PT=Portugal, $\mathrm{SI}=$ Slowenien, SK=Slowakei, UK=Großbritannien

Quelle: Schulten 2012a (Kaitz-Index); Bezzina 2012 (Niedriglohnanteil); Berechnungen der Autoren.

te Frauen im Jahre 1997 im Mittel nur 83,9 \% von Männern, so lagen die mittleren Frauenlöhne im Jahr 2006 bereits bei 89,2 \%. Damit schrumpfte der Gender Pay Gap, also die geschlechtsspezifische Einkommenslücke, innerhalb von nur neun Jahren von 16,1 auf 10,8 \% (LPC 2007, S. 134).

Eine hohe Tarifbindung trägt deutlich stärker zur Verringerung der Ungleichheit bei als Mindestlöhne. Abbildung 3 zeigt mit einer Korrelation von -0,77 einen starken Zusammenhang. Dies verwundert nicht, da in Tarifverträgen gestaffelte Entgeltgruppen, die sich als stabile Institutionalisierung von Lohnkonturen beschreiben lassen, ausgehandelt werden, die in der Regel über den Mindestlöhnen liegen und bis in obere Verdienstgruppen hineinreichen. Dennoch liegen die Umverteilungswirkungen von Tarifverträgen eher im unteren und mittleren Bereich der Einkommensverteilung, da die höheren Einkommen in den meisten Ländern häufiger einzelvertraglich vereinbart werden und die gewerkschaftliche Tarifpolitik sich vorrangig an den Interessen der Durchschnittsbeschäftigten orientiert. Dies kann man auch daran erkennen, dass die Tarifbindung mit der Relation des zweitobersten und untersten Dezils der Einkommensverteilung (P90/P10) mit-0,65 zwar noch stark, aber schwächer als mit dem Niedriglohnanteil an allen

In diese und in die folgenden Abbildungen wurden jeweils diejenigen EU-Länder einbezogen, für die entsprechende Angaben vorlagen. 
ABB.

Tarifbindung der Beschäftigten 2008/2009 und Niedriglohnanteil 2010

Angaben in Prozent

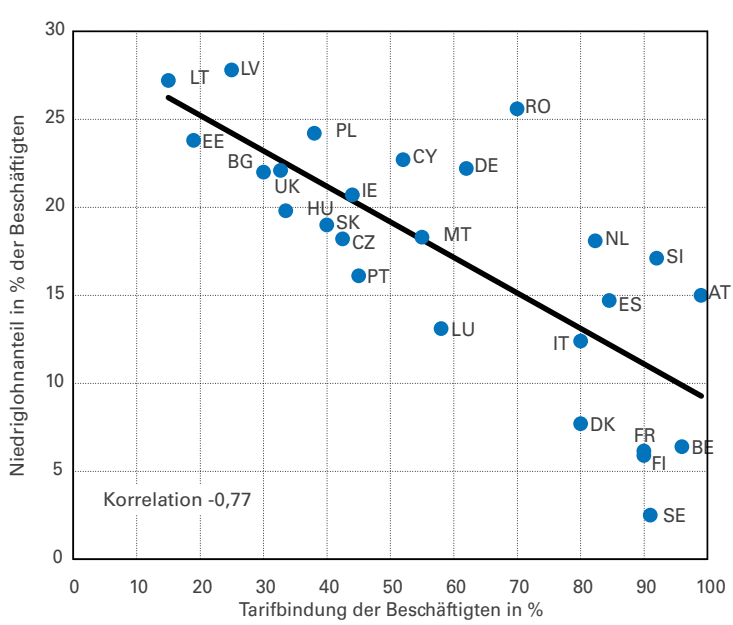

$\mathrm{AT}=$ Österreich, $\mathrm{BE}=$ Belgien, $\mathrm{BG}=\mathrm{Bulgarien}, \mathrm{CY}=\mathrm{Zypern}, \mathrm{CZ}=\mathrm{T}$ schechische Republik, DE=Deutschland, DK=Dänemark, EE=Estland, ES=Spanien, Fl=Finnland, $F R=$ Frankreich, HU=Ungarn, IE=Irland, IT=Italien, LT=Litauen, LU=Luxemburg, $\mathrm{LV}=$ Lettland, MT=Malta, NL=Niederlande, $\mathrm{PL}=$ Polen, $\mathrm{PT}=$ Portugal, RO=Rumänien, $\mathrm{SE}=\mathrm{Sch}$ weden, $\mathrm{SI}=$ Slowenien, $\mathrm{SK}=$ Slowakei, $\mathrm{UK}=\mathrm{Großb}$ ritannien

Quelle: Visser 2011 (Tarifbindung); Bezzina 2012

Mitteilungen (Niedriglohnanteil); Berechnungen der Autoren.

Vollzeitbeschäftigten korreliert.

Allerdings lässt sich kein linearer Zusammenhang zwischen Tarifbindung und Lohnungleichheit feststellen, da sich die Höhe der Tariflöhne vor allem zwischen den Branchen und Qualifikationsgruppen stark unterscheiden kann. Männer und Beschäftigte mit einem unbefristeten Vertrag profitieren stärker von einer hohen Tarifbindung als Frauen und Beschäftigte mit einem befristeten Vertrag. Befristet Beschäftigte und Leiharbeitskräfte erhalten oft nicht den gleichen Lohn oder werden niedriger eingestuft als Stammbeschäftigte. Frauen sind eher in Wirtschaftsbereichen mit geringer Tarifbindung beschäftigt. Hinzu kommt, dass nur in wenigen Ländern eine solidarische Lohnpolitik wie in den skandinavischen Ländern verfolgt wurde, die Lohnunterschiede zwischen typischen Männer- und Frauenbranchen systematisch reduzierte. Das Extrembeispiel für eine traditionelle Tarifpolitik, die hohe Geschlechterunterschiede toleriert, ist Österreich, das mit einer fast $100 \%$ igen Tarifbindung mit 24,8 \% einen weit überdurchschnittlichen Niedriglohnanteil bei Frauen hat (Männer: $8,2 \%)$.

Zu vergleichbaren Ergebnissen ist die Forschung in anderen Ländern gekommen. In ihrem Überblick über 49 Studien zu Tarifverträgen und Lohnungleichheit in den letzten Jahrzehnten sowohl in entwickelten als auch in sich entwickelnden Ländern zeigen Hayter/Weinberg (2011), dass durch Tarifverträge die Lohnungleichheit in der gesamten Wirtschaft verringert wird. Zwar können sich durch erfolgreiche Tarifverhandlungen die Lohnunterschiede zwischen Sektoren mit und ohne Tarifbindung erhöhen. Dies wird aber durch eine Verringerung der Lohnunterschiede innerhalb der Sektoren mit Tarifbindung aufgewogen. Als Zwischenergebnis können wir festhalten, dass Mindestlöhne die Löhne am unteren Ende der Lohnverteilung komprimieren, während Tarifverträge durch ihre qualifikations- und tätigkeitsorientierten Entgeltgruppen eigentlich erst die lohnpolitische Mittelschicht schaffen. Die Auswirkungen auf die interpersonelle Verteilung sind unterschiedlich. Frauen und wahrscheinlich auch andere lohnpolitisch benachteiligte Gruppen wie Migrantinnen und Migranten profitieren stärker als Männer von Mindestlöhnen. Männer wiederum haben den relativ größten Vorteil von Tarifverträgen, es sei denn, es wurde über eine solidarische Lohnpolitik erfolgreich Gleichstellungspolitik betrieben, wie dies in den skandinavischen Ländern der Fall ist. Zu beachten bleibt, dass der „Gleichstellungseffekt “ von Tarifverträgen auch für Frauen stärker ist als der von Mindestlöhnen.

\section{Niedriglöhne und die Gesamt- architektur von Lohnsystemen}

Während die isolierten Wirkungen von Mindestlöhnen und Tarifverträgen auf die Lohnverteilung gut untersucht sind, sind die Interaktionen zwischen diesen Institutionen bislang kaum thematisiert worden. Im Folgenden gehen wir dieser Frage nach. Abbildung 4 zeigt eine allerdings nur schwach positive Relation von Mindestlöhnen und Tarifbindung (Korrelation 0,37), was zwar auf leicht komplementäre Beziehungen, aber auch auf starke Länderunterschiede hinweist. In Ländern mit hoher Tarifbindung und größerer Verhandlungsstärke der Gewerkschaften färben Tarifverträge auf Mindestlöhne ab; und dort, wo die Tarifbindung schwach ist, erreichen die Mindestlöhne eher ein geringes Niveau. Grimshaw/Rubery (2013) weisen allerdings darauf hin, dass zwischen 1995 und 2006 die Mindestlöhne in Ländern mit geringer Tarifbindung stärker erhöht wurden als in Ländern mit hoher Tarifbindung. Wenn die Mindestlöhne zu sehr abgesunken sind, kann sich allgemeiner politischer Druck aufbauen, sie zu erhöhen. Bei hoher Tarifbindung existieren offensichtlich durch eine entwickelte Sozialpartnerschaft wirkungsvolle institutionalisierte Kanäle einer schrittweisen Anpassung auch der unteren Löhne.

$\mathrm{Zu}$ beachten sind auch die Länder, die aus unterschiedlichen Gründen keinen gesetzlichen Mindestlohn haben: wie die skandinavischen Länder, Österreich und Deutschland. Auch die bewusste Ablehnung oder Nicht-Einführung eines Mindestlohns ist eine Interaktion, die sich aus vermuteten negativen Wechselwirkungen zwischen gesetzlichen Mindestlöhnen und Tarifverträgen ergibt. 
Tarifbindung, Organisationsgrad der Arbeitgeber und Beschäftigten und Niedriglohnanteile in Ländern

Angaben in Prozent

\begin{tabular}{|c|c|c|c|c|c|c|}
\hline & $\begin{array}{l}\text { Tarifbindung der } \\
\text { Beschäftigten } \\
\text { (2008/09) } \\
\text { in \% }\end{array}$ & $\begin{array}{c}\text { Gewerkschaftsmit- } \\
\text { glieder (2008/09) } \\
\text { in \% der } \\
\text { Beschäftigten }\end{array}$ & $\begin{array}{c}\text { Organisationsgrad } \\
\text { der Arbeitgeber (2006) } \\
\text { in \% der } \\
\text { Beschäftigten }\end{array}$ & $\begin{array}{c}\text { Anteil allgemein- } \\
\text { verbindlicher } \\
\text { Tarifverträge }\end{array}$ & $\begin{array}{c}\text { Niedriglohn- } \\
\text { anteil (2010) } \\
\text { in \% der } \\
\text { Beschäftigten }\end{array}$ & $\begin{array}{c}\text { Mindestlohnhöhe } \\
\text { (Kaitz-Index 2010) } \\
\text { in \% des Medians } \\
\text { (Vollzeit) }\end{array}$ \\
\hline Belgien & 96 & 52 & 72 & Sehr hoch & 6,4 & 52 \\
\hline Dänemark & 80 & 64,5 & 52 & - & 7,7 & - \\
\hline Deutschland & 59 & 18,6 & 63 & Gering & 22,2 & - \\
\hline Frankreich & 90 & 7,6 & 78 & Sehr hoch & 6,1 & 60 \\
\hline Niederlande & 82 & 19 & 85 & Hoch & 18,1 & 47 \\
\hline Osterreich & 99 & 35 & 100 & - & 15,0 & - \\
\hline Schweden & 91 & 69 & 55 & - & 2,5 & - \\
\hline Spanien & 84,5 & 17 & 72 & Hoch & 14,7 & 44 \\
\hline Ungarn & 34 & 16,8 & 40 & - & 19,8 & 47 \\
\hline $\begin{array}{l}\text { Vereinigtes } \\
\text { Königreich }\end{array}$ & 33 & 27,5 & 40 & - & 22,1 & 46 \\
\hline
\end{tabular}

\section{ABB. 4}

\section{Tarifbindung der Beschäftigten 2008/2009 und relative Höhe des Mindestlohns (Kaitz- \\ Index) 2010}

Angaben in Prozent

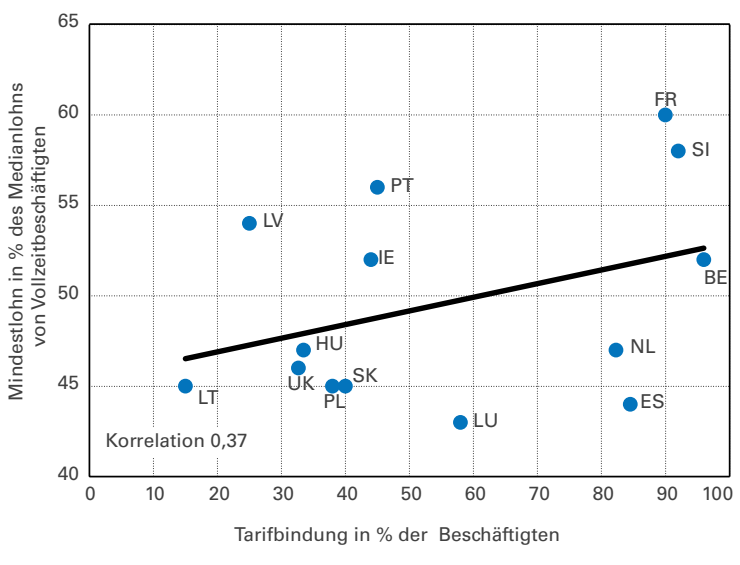

$\mathrm{BE}=$ Belgien, ES=Spanien, FR=Frankreich, $\mathrm{HU}=$ Ungarn, $\mathrm{IE}=\mathrm{Irland}, \mathrm{LT}=\mathrm{Litauen}$ $\mathrm{LU}=$ Luxemburg, LV=Lettland, NL=Niederlande, $\mathrm{PL}=$ Polen, $\mathrm{PT}=$ Portugal, Sl=Slowenien, SK=Slowakei, UK=Großbritannien

Quelle: Schulten 2012a (Kaitz-Index): Visser 2011 (Tarifbindung); Berechnungen der Autoren.

Auf der Basis von mehreren vergleichenden Länderstudien, ${ }^{2}$ an denen wir in den letzten Jahren beteiligt waren, lassen sich verschiedene Typen nach der Gesamtarchitektur ihrer Lohnsysteme unterscheiden. In Tabelle 1 sind für die zehn ausgewählten EU-Staaten, auf die sich die Typologie bezieht, einige zentrale Indikatoren $\mathrm{zu}$ den industriellen Beziehungen und Merkmalen der Lohnsysteme zusammengestellt.

\subsection{Typologie der Interaktionen}

Wir unterscheiden zwischen sechs Typen, wobei bereits vorab darauf hinzuweisen ist, dass es innerhalb der Länder zwischen verschiedenen Branchen bzw. Sektoren durchaus unterschiedliche Ausprägungen geben kann:

(1) Die direkte Interaktion: Der Mindestlohn erreicht in diesem Typ ein relativ hohes Niveau und der Abstand zu den untersten Tarifgruppen ist in den meisten Branchen nur gering. Die Gewerkschaften sind zu schwach, um ohne Hilfe des Staates in autonomen Tarifverhandlungen die untersten Lohngruppen über das Niveau des Mindest-

2 Zu nennen sind ein von der Hans-Böckler-Stiftung gefördertes Projekt zu Lohnstandards im Baugewerbe im internationalen Vergleich (Bosch et al. 2011) und die Evaluation der branchenbezogenen Mindestlohnregelungen in Deutschland (Bosch/Weinkopf 2012a). Besonders herauszuheben ist das EU-Projekt „Minimum Wage Systems and Changing Industrial Relations", an dem auch Teams aus Großbritannien, Kroatien, Spanien und Ungarn beteiligt waren. Eine erste Version der Ländertypologie ist in Bosch et al. (2011) enthalten und wurde vor allem im Kontext einer aktuellen Buchpublikation (Grimshaw 2013) weiter verfeinert. Wir haben sie hier um einige weitere Länder ergänzt und leicht modifiziert. 
lohnes anzuheben. Jede Erhöhung der Mindestlöhne drückt damit das gesamte Tarifgefüge nach oben und die rippleEffekte sind sehr hoch. Ein Beispiel hierfür ist Frankreich. Der hohe Anteil der Beschäftigten, die trotz der sehr hohen Tarifbindung auf dem Niveau des Mindestlohns bezahlt werden, deutet auf die enge Koppelung hin (Caroli/Gautié 2008). Die Folge ist, dass der Mindestlohn im Mittelpunkt der politischen Aufmerksamkeit steht und die Tarifpolitik mitzieht. Die Koppelung von Mindestlöhnen und Tarifverträgen wird durch die Allgemeinverbindlicherklärung (AVE) der Entgeltgruppen und Tariflöhne institutionalisiert. Überdies hat der französische Staat in den letzten Jahren starke finanzielle Anreize gesetzt, alle tariflichen Lohngruppen über den gesetzlichen Mindestlohn anzuheben. ${ }^{3}$ In Abbildung 5 wird dies durch das direkte Aufsetzen der Tariflöhne auf das Niveau des gesetzlichen Mindestlohns veranschaulicht.

(2) Die distanzierte Koexistenz: In diesem Typ liegen die Tariflöhne aufgrund einer Tradition autonomer Tarifpolitik und starker Sozialpartner überwiegend deutlich über dem Mindestlohn und die Tarifverhandlungen werden nicht direkt durch Anhebungen der Mindestlöhne bestimmt. Belgien und Spanien (Banyuls et al. 2010) verdanken ihre unterdurchschnittlichen Niedriglohnanteile (6,1 bzw. 14,7 \% im Jahr 2010 - vgl. Tabelle 1) weniger ihrem Mindestlohn als vielmehr der hohen Tarifbindung mit kollektiv ausgehandelten Löhnen. In Belgien kommt hinzu, dass der Mindestlohn mit einem Kaitz-Index von $52 \%$ auch vergleichsweise hoch ist. Die Vorrangposition der Tarifpolitik wird in diesen Ländern durch fast flächendeckende Allgemeinverbindlicherklärungen gestützt (Schulten 2012b). Dies gilt auch für die Niederlande, wobei hier der Anteil der Niedriglohnbeschäftigten mit gut $18 \%$ allerdings sogar über dem Durchschnitt der EU27-Staaten liegt. Ursächlich hierfür dürften vor allem die deutlich niedrigeren Mindestlöhne für jüngere Beschäftigte sein. Mindestlöhne dienen bei diesem Typ vor allem dem Schutz der Tariflöhne vor einem Abrutschen nach unten. Da die Tariflöhne meist über dem Mindestlohn liegen, hängen die Tariferhöhungen in den meisten Branchen nicht direkt von Erhöhungen der Mindestlöhne ab. Es kann sogar zu einer Vernachlässigung des Mindestlohns kommen wie in der Vergangenheit in Spanien, wo Politik und Gewerkschaften wegen der hohen Tarifbindung keinen Druck verspürten, sich auf eine Anhebung der Mindestlöhne zu konzentrieren. In Abbildung 5 wird dies am Beispiel von Belgien dadurch gekennzeichnet, dass es eine erkennbare Distanz zwischen den Mindest- und den untersten Tariflöhnen gibt.

(3) Der isolierte Mindestlohn: In diesem Typ ist die Tarifbindung gering. Nur in einzelnen Branchen gelingt es Gewerkschaften und Arbeitgebern, auf Branchenebene Tarifverträge auszuhandeln, die auf dem Mindestlohn aufsetzen. Die Wirkungen sind jedoch nicht systematisch und durch die geringe Verbreitung von Tarifverträgen eher begrenzt.
Über die Höhe der ripple-Effekte wird auf betrieblicher Ebene entschieden. Angesichts der Schwäche der Gewerkschaften in der Privatwirtschaft kommt es zu einseitigen Entscheidungen der Unternehmen, die fast ausschließlich von Effizienzüberlegungen und der Arbeitsmarktsituation abhängen. Bei hoher Verfügbarkeit von Arbeitskräften und hoher Austauschbarkeit der Arbeitskräfte können die ripp$l e$-Effekte auch negativ sein. Beispiele für diesen Typ sind Großbritannien und auch Ungarn, das allerdings aufgrund der Differenzierung der Mindestlöhne nach Qualifikation hier einem eigenen Typ zugeordnet wird. Aufgrund der Schwäche der Tarifpolitik konzentrieren sich Gewerkschaften und Politik auf die Anhebung der Mindestlöhne (Grimshaw et al. 2010).

(4) Extensiver Mindestlohn: Wenn die Tarifpartner schwach sind und die Tarifbindung gering ist, aber die Regierung dennoch tarifähnliche Entgeltstrukturen durchsetzen will, kann sie den Mindestlohn differenzieren. Ungarn ist ein gutes Beispiel für diesen Typ. Es wurden drei unterschiedliche Mindestlöhne eingeführt, eine unterste Rate für Anund Ungelernte, eine mittlere für Qualifizierte und eine noch höhere für Beschäftigte mit einem akademischen $\mathrm{Ab}$ schluss. Der oberste Mindestlohn wurde allerdings im Jahr 2009 wieder abgeschafft und hat nur noch empfehlenden Charakter. Branchenstudien zeigen, dass mit den beiden Mindestlöhnen ein Basisgerüst für tarifliche und betriebliche Lohnstrukturen geschaffen wurde, das negative rippleEffekte für Qualifizierte einschränkte (Neumann 2010). Die Unterschiede zum Typ 3 sind eher graduell und beziehen sich vor allem auf das Regierungshandeln.

(5) Autonome Tarifpolitik: In Ländern mit starker Tarifbindung und großer autonomer Handlungsmacht der Gewerkschaften werden gesetzliche Mindestlöhne als Eingriff in die Tarifpolitik abgelehnt und tarifpolitische Substitute geschaffen. Durch die fast lückenlose Tarifbindung wirken die untersten tariflichen Lohngruppen wie Mindestlöhne. Beispiele für diesen Typ sind Dänemark und Schweden, wenn-

3 Die französische Regierung beschloss im Jahr 2008 mit ihrem Gesetz zur "Conditionnalité des allègements de charges SMIC", die Tariflöhne auf das Niveau des SMIC anzuheben. Nach diesem Gesetz werden die erheblichen $\mathrm{Zu}$ schüsse zu den Arbeitgeberbeiträgen zur Sozialversicherung zunächst auf der Basis derTariflöhne berechnet. Wenn die Tariflöhne unterhalb des SMIC liegen, sinken damit die Zuschüsse. Wenn innerhalb von drei Jahren die Tariflöhne nicht auf das Niveau des SMIC angehoben werden, entfallen die Zuschüsse ganz. Das Gesetz trat nach einer Verschiebung zum Jahresbeginn 2013 in Kraft. Die Androhung der Streichung beachtlicher Lohnzuschüsse hat bereits im Vorfeld zu einer Beschleunigung der zuvor stagnierenden Lohnverhandlungen geführt. Die Regierung verfolgt das Ziel, dass dieTariflöhne künftig flächendeckend über den SMIC angehoben werden (Ministère du Travail, de I'Emploi, de la Formation Professionelle et du Dialogue Social 2013). 


\section{Typologie der Wechselwirkungen zwischen Tarifverträgen}

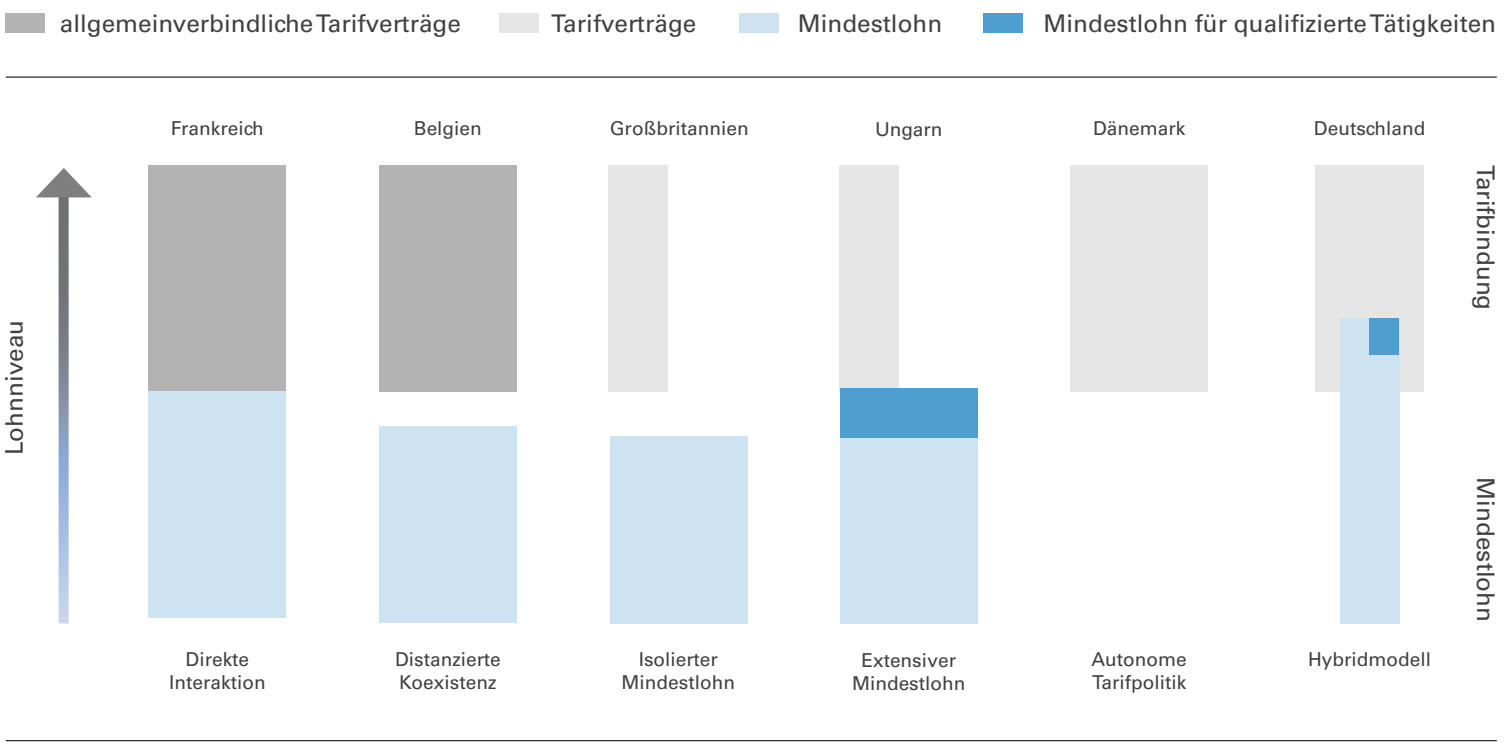

gleich die umfassende Tarifbindung nach einigen Urteilen des Europäischen Gerichtshofs (EuGH) (Laval, Viking) mittlerweile durch grenzüberschreitende Entsendungen an den Rändern Risse zu verzeichnen hat (Bosch 2013). In Dänemark und Österreich haben die Tarifpartner einen tariflichen Mindestlohn vereinbart. In Dänemark garantiert der hohe gewerkschaftliche Organisationsgrad die Durchsetzung dieses Mindestlohnes, während in Österreich die Zwangsmitgliedschaft der Arbeitgeber in der Wirtschaftskammer für eine sehr hohe Tarifbindung sorgt. Allerdings liegt der Niedriglohnanteil in Österreich bei immerhin $15 \%$, was darauf verweist, dass ein nicht unerheblicher Teil der Tariflöhne unterhalb der Niedriglohnschwelle liegt, während dies in Schweden offenbar nur sehr selten der Fall ist (2,5\% Niedriglohnbeschäftigte). In diesen Ländern wird kein Bedarf gesehen, gesetzliche Mindestlöhne einzuführen (vgl. für die skandinavischen Länder Eldring/Alsos 2012). In Abbildung 5 spiegelt sich das darin wider, dass es bei Dänemark als Beispiel für diesen Typ lediglich einen breiten Block mit geringer Streuung nach unten für die Tariflöhne gibt.

(6) Hybridmodell: Dieser Typ ist eine Kombination unterschiedlicher Modelle. Dominant sind Tarifverträge, die allerdings für deutlich weniger Beschäftigte gelten als in den Typen 1, 2 und 5. Da die Tarifpolitik nicht durch einen generellen Mindestlohn gestärkt wird, finden sich in Bereichen mit geringer Verhandlungsmacht Tariflöhne, die weit unterhalb der Niedriglohnschwelle liegen. In einzelnen Branchen wurden Mindestlöhne vereinbart, die zum Teil auch eine höhere Bezahlung für Fachkräfte vorsehen, was dem Typ 4 entspricht. Das Referenzbeispiel hierfür ist
Deutschland, auf das wir in Abschnitt 4 noch detaillierter eingehen. Die Darstellung von Deutschland in Abbildung 5 soll veranschaulichen, dass die Tarifbindung im mittleren Bereich liegt, es aber auch in größerem Umfang tarifliche Niedriglöhne gibt. Gleichzeitig liegen einige der branchenbezogenen Mindestlöhne deutlich über der Niedriglohnschwelle und in manchen Branchen existieren zusätzliche höhere Mindestlöhne für qualifizierte Tätigkeiten (vgl. auch Abbildung 7). ${ }^{4}$

\subsection{Einfluss der Lohninstitutionen}

Die einzelnen Typen sind durch unterschiedliche Konstellationen relevanter Lohninstitutionen gekennzeichnet, auf die wir nun unter Bezug auf die in Tabelle 1 ausgewiesenen Indikatoren für die zehn ausgewählten Länder genauer eingehen.

In sieben dieser Länder sorgt das institutionelle Gefüge für eine hohe und stabile Tarifbindung von über $80 \%$. Die dahinter stehenden institutionellen Konstellationen sind allerdings sehr unterschiedlich. In den Niederlanden, Spanien und vor allem in Frankreich resultiert die hohe Tarifbindung nicht aus einem hohen gewerkschaftlichen Organisationsgrad der Beschäftigten, sondern aus allgemeinverbindlichen Tarifverträgen und einer hohen Organisationsquote von Unternehmern, die nicht zufällig ist. Da der Staat die Tarifpolitik in diesen Ländern, wie auch in Belgien, über All-

4 Bei der relativen Höhe der Mindestlöhne ist zu beachten, dass die Medianlöhne von Vollzeitbeschäftigten für Ostund Westdeutschland getrennt berechnet wurden. 
gemeinverbindlicherklärungen (AVE) aktiv fördert, sind die Unternehmen daran interessiert, über starke Arbeitgeberverbände an diesen politischen Prozessen mitzuwirken. Belgien, Frankreich und die Niederlande haben de facto zwei Arten von Mindestlöhnen: den gesetzlichen Mindestlohn als Untergrenze und die allgemeinverbindlichen Tariflöhne, die sich über das gesamte Lohngitter erstrecken. Die Tarifbindung in Frankreich und den Niederlanden hat durch die positiven Wechselwirkungen zwischen den unterschiedlichen Lohninstitutionen trotz abnehmender Gewerkschaftsmitgliedschaft der Beschäftigten in den vergangenen Jahren sogar noch zugenommen (Bosch et al. 2010, S. 95).

In Dänemark und Schweden ist die hohe Tarifbindung demgegenüber vor allem Folge des hohen gewerkschaftlichen Organisationsgrades der Beschäftigten, der wie in Belgien durch das Ghent-System ${ }^{5}$ stabilisiert wird. In Dänemark haben Arbeitgeberverbände und Gewerkschaften zudem einen tariflichen Mindestlohn vereinbart, dessen Einhaltung von den Sozialpartnern ohne staatliche Hilfe wirkungsvoll kontrolliert wird (Westergaard-Nielsen 2008). In Österreich wird die flächendeckende Tarifbindung dadurch erreicht, dass die Verbandsmitgliedschaft von Arbeitgebern obligatorisch ist.

Bemerkenswert ist jedoch, dass sich die Länder trotz der durchgängig sehr hohen Tarifbindung hinsichtlich der Niedriglohnquote deutlich unterscheiden. Während in Dänemark, Frankreich, Belgien und insbesondere in Schweden nur ein kleiner Teil der Beschäftigten unterhalb der Niedriglohnschwelle bezahlt wird, sind es in Österreich $15 \%$ und in den Niederlanden sogar 18,1 \% der Beschäftigten. Die

ABB.

\section{Niedriglohnanteile nach Wirtschaftsgruppen in Deutschland 2010}

Angaben in Prozent aller Beschäftigten

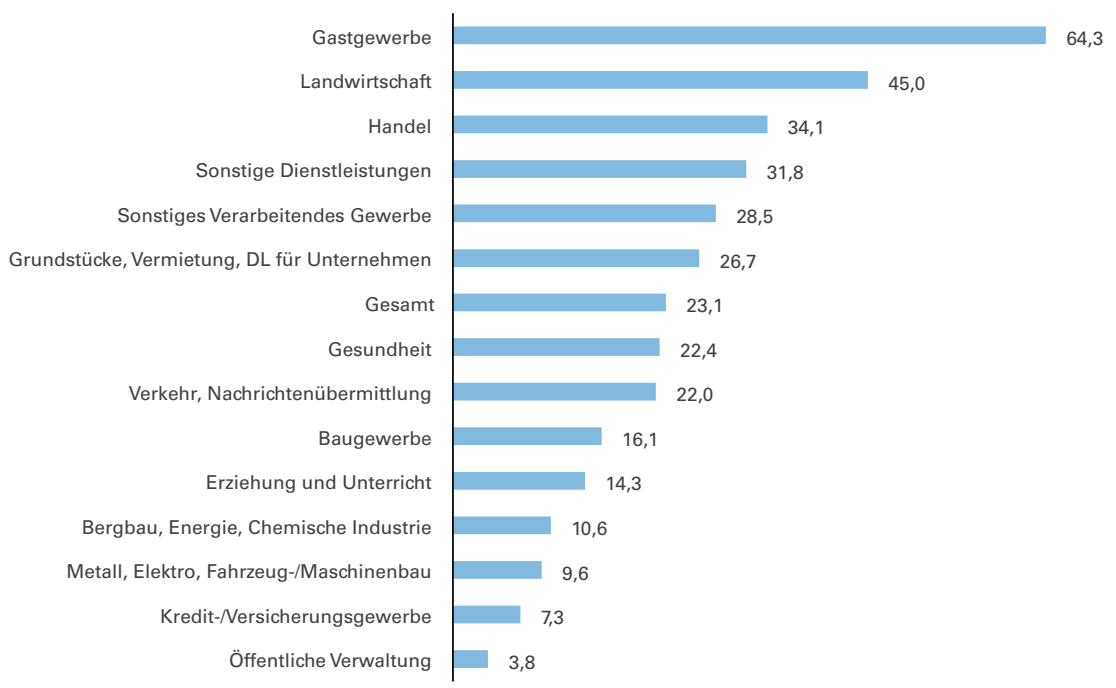

Quelle: SOEP (2010); IAQ-Berechnungen (Thorsten Kalina). geringere tarifliche Bewertung von Frauentätigkeiten in beiden Ländern und die niedrigeren Mindestlöhne für Jugendliche in den Niederlanden sind wichtige Ursachen hierfür. In den drei Ländern mit geringerer Tarifbindung liegen die Niedriglohnanteile jedoch noch höher, was die beschriebenen Gleichheitseffekte von Tarifverträgen bestätigt.

In Großbritannien und Ungarn ist jeweils nur ein Drittel der Beschäftigten tarifgebunden. Tarifvereinbarungen werden (mit Ausnahme des öffentlichen Sektors) fast nur auf Unternehmensebene abgeschlossen. Die Flächentarifverträge, die in Großbritannien in den 1980er Jahren noch weitgehend die Bezahlung regelten, haben den Lohnwettbewerb der letzten Jahrzehnte nicht überlebt. AVE gelten als marktfremde Eingriffe in die Koalitionsfreiheit. Da die Arbeitgeber in beiden Ländern wenig Notwendigkeit sehen, sich in Arbeitgeberverbänden zum Zwecke von Tarifverhandlungen zu organisieren, hängt die Tarifbindung allein von der Stärke der Gewerkschaften ab. Tarifverträge gelten fast nur für Gewerkschaftsmitglieder und entfalten darüber hinaus kaum lohnpolitische Wirkungen. Obwohl beide Länder gesetzliche Mindestlöhne haben, arbeitet etwa jede und jeder fünfte Beschäftigte für einen Niedriglohn. Anders als in Deutschland werden mit den Mindestlöhnen aber zumindest verbindliche Lohnuntergrenzen gezogen, die legal in keiner Branche unterschritten werden können.

In Deutschland ist die Tarifbindung der Beschäftigten von einstmals über $80 \%$ in Westdeutschland auf $59 \%$ bundesweit im Jahr 2011 gesunken (61 \% in West- und $49 \%$ in Ostdeutschland, vgl. Ellguth/Kohaut 2012, S. 298), vor allem durch Austritte aus Arbeitgeberverbänden oder Nichteintritte von neu gegründeten Unternehmen. Die Tarifbindung ist zwar deutlich höher als in Großbritannien oder Ungarn, aber aufgrund des Verzichts auf einen gesetzlichen Mindestlohn haben sich neben dem klassischen deutschen Tarifmodell große weiße Zonen eines unregulierten Arbeitsmarktes herausgebildet, in dem sich Niedriglöhne ausgebreitet haben. Zur Kostenersparnis haben viele tarifgebundene Unternehmen Tätigkeiten in kleinere und mittlere Unternehmen ausgelagert, die keinem Tarifvertrag unterliegen oder niedrigere tarifliche Standards haben. In diesen Unternehmen gibt es meist keine Betriebsräte, die mit ihren starken Mitbestimmungsrechten die wichtigste Kontrollinstanz zur Einhaltung tariflicher Lohnstandards in Deutschland sind. In einzelnen Dienstleistungsbranchen mit niedriger Organisationsquote sowohl der Unternehmer als auch der Beschäftigten, wie z. B. im Einzelhandel, wurden in der Vergangenheit tarifliche Standards in den vielen kleinen und mittleren Unternehmen

5 Als Ghent-System bezeichnet man Wohlfahrtssysteme, deren Verwaltung und Steuerung weitgehend an die Gewerkschaften übertragen ist. Das gilt für die Arbeitslosenversicherungen in Belgien, Dänemark, Finnland, Schweden und Island. Da Zugang zur Arbeitslosenversicherung die Mitgliedschaft in einer Gewerkschaft erfordert, ist die gewerkschaftliche Organisationsquote in diesen Ländern überdurchschnittlich hoch. 
vorrangig durch eine AVE gesichert. Seit mehreren Jahren verweigert aber die Bundesvereinigung der Arbeitgeberverbände (BDA) zunehmend ihre Zustimmung hierzu (Bispinck 2012). Die Tarifinstitutionen sind durch das fast uneingeschränkte Lohndumping in höchstem Maße instabil geworden, was auch dazu beigetragen hat, dass Deutschland unter den hier betrachteten Ländern inzwischen mit 22,2 \% den höchsten Anteil von Niedriglohnbeschäftigten aufweist. ${ }^{6}$

\section{Unterschiedliche Lohninstitutionen nach Branchen und Regionen in Deutschland}

Typologien sind ein wichtiges Hilfsmittel zum Verständnis der dominanten Strukturen in unterschiedlichen Ländern und unverzichtbar für den Ländervergleich. Allerdings sind sie hochaggregiert und vernachlässigen, dass sich die institutionellen Arrangements zur Lohnfindung auch innerhalb einzelner Länder je nach Branche und Region deutlich voneinander unterscheiden können. Besonders ausgeprägt ist die Heterogenität von Lohninstitutionen in Deutschland, das deshalb inzwischen einen eigenen Ländertyp konstituiert. Bis 1990 hätte man Deutschland wegen seiner damals hohen Tarifbindung noch dem Typ „Autonome Tarifpolitik“ zuordnen können. Mindestlöhne wurden von Gewerkschaften und Unternehmensverbänden einvernehmlich als unzulässiger Eingriff in die Tarifautonomie abgelehnt. In großen Teilen des verarbeitenden Gewerbes, im öffentlichen Dienst und im Bereich der Banken und Versicherungen ist die Tarifbindung auch heute noch hoch. Vor allem bei den privaten Dienstleistungen und zum Teil auch in kleineren Betrieben des verarbeitenden Gewerbes (zum Beispiel im Handwerk) haben sich inzwischen jedoch große tariffreie Zonen mit nur kleinen Inseln tarifgebundener Betriebe herausgebildet, in denen der Anteil der gering bezahlten Beschäftigten rasch gestiegen ist (Abbildung 6).

Vor dem Hintergrund dieser Entwicklung haben die Gewerkschaften und auch einige Arbeitgeberverbände ihre Haltung zum Mindestlohn revidiert. Aktuell gelten in elf Branchen (Stand Februar 2013) tarifliche Mindestlöhne, die von den Sozialpartnern ausgehandelt und von der Regierung nach dem Arbeitnehmer-Entsendegesetz auch für entsandte Beschäftigte aus anderen Ländern für allgemeinverbindlich erklärt worden sind. ${ }^{7}$ Diese Form der Allgemeinverbindlichkeit hat einen stärkeren Charakter, da der Staat auch die Einhaltung der Mindestlöhne kontrolliert und Sanktionen verhängen kann, während bei der traditionellen AVE nach dem Tarifvertragsgesetz die Tarifpartner selbst für die Durchsetzung sorgen müssen.

In acht dieser Branchen sind die Wirkungen der Mindestlöhne im Jahr 2011 evaluiert worden. Dabei haben sich große Unterschiede in den Wechselwirkungen von Mindest-
ABB. 7

\section{Relative Höhe der branchenbezogenen Mindestlöhne in Ost- und Westdeutschland (Februar 2013)}

Angaben in Prozent des jeweiligen Medians von Vollzeitbeschäftigten 2011 West Ost

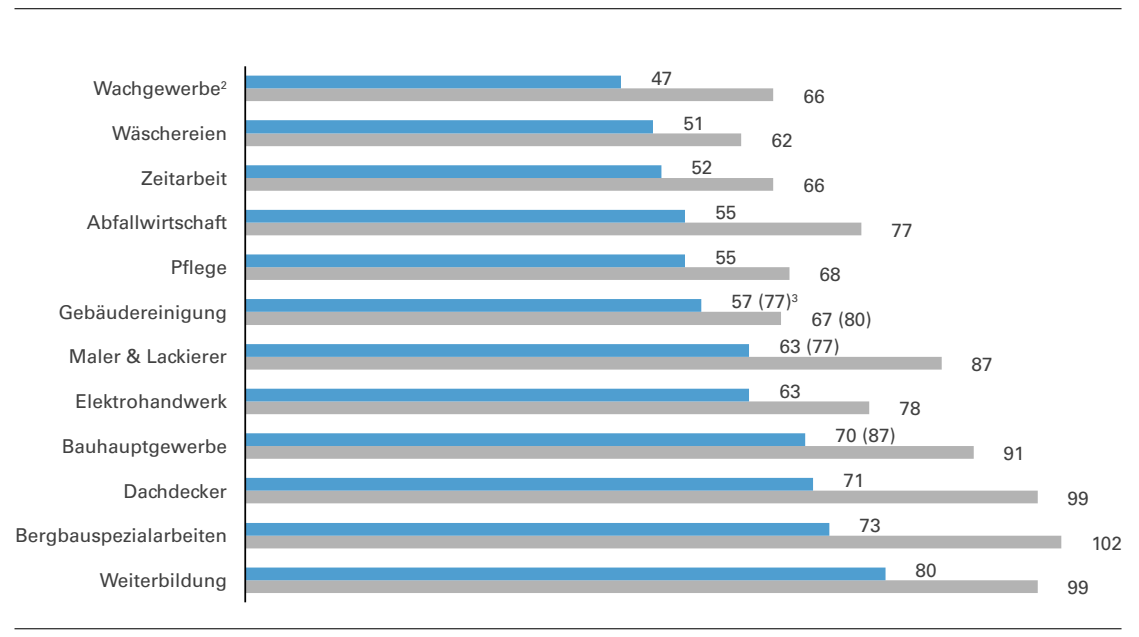

'Der Median der Stundenlöhne von Vollzeitbeschäftigten lag nach IAQ-Berechnungen auf der Basis des SOEP im Jahr 2011 in Westdeutschland bei 15,81 € und in Ostdeutschland bei 11,32 €

${ }_{2} \mathrm{Im}$ Wachgewerbe liegen die nach Bundesländern differenzierten Mindestlöhne seit Anfang 2013 zwischen 7,50€ (Ostdeutschland sowie Berlin, Rheinland-Pfalz, Saarland, Schleswig-Holstein) und 8,90 € (Baden-Württemberg), was $56 \%$ deutschland sowie Berlin, Rheinland-Pfalz

${ }^{3}$ Die Werte in Klammern beziehen sich auf den höheren Mindestlohn für qualifizierte Tätigkeiten.

Quelle: Zusammenstellung und Berechnung der Autoren.

und Tariflöhnen gezeigt. Hinsichtlich der formellen Institutionen überwiegen die Ähnlichkeiten. Die Mindestlöhne wurden zwischen den Sozialpartnern ausgehandelt und entsprechen meist den unteren Lohngruppen in einem umfassenderen Tarifgefüge. In einigen Handwerksbranchen (Bauhauptgewerbe, Gebäudereinigung, Maler) wurde sogar ein zweiter Mindestlohn für qualifiziertere Kräfte vereinbart, der aber in Ostdeutschland im Bauhauptgewerbe und bei den Malern aufgrund geringer Durchsetzungschancen zwischenzeitlich wieder abgeschafft worden ist. In Branchen mit konkurrierenden Tarifverträgen (Pflege, Abfallwirtschaft, Wäschereien) liegen die Mindestlöhne meist unterhalb der niedrigsten Tariflöhne und sollen vor allem die Lohnkonkurrenz durch nicht tarifgebundene Unternehmen begrenzen.

Deutliche Unterschiede zwischen den Branchen bestehen auch hinsichtlich des Anteils der Beschäftigten, die auf dem Niveau der Mindestlöhne bezahlt werden. Im Reinigungsgewerbe konzentrieren sich die meisten Beschäftigten auf dem Niveau der unteren Mindestlohngruppe und es gibt aufgrund der kurzen Anlernzeiten, des geringen

6 Noch höhere Niedriglohnanteile in der EU hatten im Jahr 2010 nur Estland und Zypern (jeweils 23,8 \%), Lettland $(27,8 \%)$, Litauen $(27,2 \%)$ sowie Polen $(24,2 \%)$ und Rumänien (25,6 \%) (Bezzina 2012).

7 Für die Zeitarbeitsbranche wurde dies im Arbeitnehmerüberlassungsgesetz geregelt. 
gewerkschaftlichen Organisationsgrads und des ausgeprägten Preiswettbewerbs kaum betriebliche Lohndifferenzen. Der untere Mindestlohn in Ost- und Westdeutschland ist der übliche Lohn für die meisten Reinigungskräfte, was die ripple-Effekte begrenzt. Nur für die kleine Gruppe der meist männlichen Beschäftigten in der Außenreinigung gilt der höhere zweite Mindestlohn.

In anderen Branchen mit höheren Anteilen qualifizierter Beschäftigter und einer langen Tradition gewerkschaftlicher Tarifpolitik mit etablierten Normen der Lohndifferenzierung konnten, zumindest in Westdeutschland, hingegen große regionale Unterschiede festgestellt werden. Diese lassen sich so zusammenfassen (Bosch/Weinkopf 2012a):

(1) Angesichts der höheren Arbeitslosigkeit in Ostdeutschland haben die dortigen Unternehmen deutlich bessere Chancen als in Westdeutschland, qualifizierte Beschäftigte zu halten und die innerbetriebliche Motivation nicht zu gefährden, selbst wenn sie ihnen nur den Mindestlohn oder geringfügig mehr zahlen.

(2) Die Betroffenheit der ostdeutschen Beschäftigten von Mindestlöhnen ist demnach erheblich höher als in Westdeutschland, wo die Löhne nach Einführung oder Erhöhung der Mindestlöhne meist nur für einen kleinen Teil der Beschäftigten angehoben werden müssen.

(3) Die Mindestlöhne haben in Ostdeutschland zu einer Komprimierung der Löhne nahe am Mindestlohn geführt. Zum Teil ist es zu negativen ripple-Effekten durch relativ geringere Steigerungen der Löhne oberhalb des jeweiligen Mindestlohns gekommen. Die staatlichen Kontrollen sind erfolgreich bei der Durchsetzung der unteren Mindestlöhne, versagen aber bei der Durchsetzung von höheren Mindestlöhnen für Fachkräfte. Vor diesem Hintergrund haben die Gewerkschaften auf Druck der Arbeitgeber im ostdeutschen Bauhauptgewerbe in Ostdeutschland vor einigen Jahren auf diesen zweiten Mindestlohn zugunsten einer schnelleren Anhebung des unteren Mindestlohns verzichtet. Aufgrund der geringeren Tarifbindung und der Schwierigkeit, Mindest- und Tarifstandards in der Praxis durchzusetzen, gilt in Ostdeutschland in erheblich mehr Branchen als im Westen inzwischen das britische Modell eines „isolierten Mindestlohns.“

(4) In Westdeutschland hingegen ist in den meisten Branchen durch die Einführung und die Erhöhung der Mindestlöhne die gesamte Lohnstruktur nach oben geschoben worden. Diese ähneln demnach dem französischen Modell der „direkten Interaktion“, allerdings in abgeschwächter Form, da die Tariflöhne über dem Mindestlohn i.d.R. nicht allgemeinverbindlich sind und es durchaus auch zu relativen Absenkungen der höheren Tariflöhne gekommen ist.

(5) Bemerkenswert ist nicht zuletzt, dass die relative Höhe der branchenbezogenen Mindestlöhne - gemessen am
Kaitz-Index - in Deutschland z. T. deutlich über dem Niveau der gesetzlichen Mindestlöhne in anderen EU-Staaten liegt. Dies gilt insbesondere für Ostdeutschland, wo fast alle Mindestlöhne die Niedriglohnschwelle erreichen oder sie sogar teils deutlich übersteigen. In Westdeutschland liegen die relativen Mindestlöhne demgegenüber mit Ausnahme weniger Branchen etwa auf dem Niveau der gesetzlichen Mindestlöhne in anderen EU-Staaten (Abbildung 7).

\section{Zusammenfassung und Schlussfolgerungen}

Die internationale Forschung hat in den letzten Jahren in vielen Untersuchungen belegen können, dass die großen Unterschiede des Ausmaßes der Niedriglohnbeschäftigung zwischen den OECD-Ländern vor allem auf Unterschiede in den Institutionen zur Lohnfindung zurückzuführen sind. In diesem Beitrag haben wir die Wirkungen von Mindestlöhnen und unterschiedlichen Tarifsystemen auf die Lohnungleichheit untersucht. Beide Institutionen haben unterschiedliche Wirkungen, aber ihr Zusammenspiel hängt sehr stark von der Architektur des nationalen Lohnsystems ab (Grimshaw et al. 2013). Gesetzliche Mindestlöhne beeinflussen vor allem die Lohnverteilung innerhalb des Niedriglohnsektors, während Tarifverträge stärkere Auswirkungen auf die Lohnverteilung um den Medianlohn haben. Es konnte gezeigt werden, dass Mindestlöhne den Anteil der Niedriglohnbeschäftigten nur geringfügig verringern, da diese meist unterhalb der Niedriglohnschwelle liegen. Innerhalb des Niedriglohnsektors tragen Mindestlöhne aber dazu bei, das Ausfransen der Löhne nach unten zu verhindern. Tarifverträge haben eine stärkere Wirkung auf Lohnungleichheit, da sie Lohngitter mit Löhnen bis über den Medianlohn enthalten. Von ihnen profitieren „Normalbeschäftigte" mit unbefristetem Vertrag und Männer überdurchschnittlich. Der Zusammenhang zwischen der Höhe der Mindestlöhne und der Tarifbindung ist eher gering. Mindestlöhne können auch zu Erhöhungen der Löhne oberhalb des Mindestlohnes führen, die in der Literatur als „ripple-Effekte“ bezeichnet werden. Hintergrund kann u. a. sein, dass Unternehmen ein Interesse haben, bei Anhebungen von Mindestlöhnen die alten Lohnunterschiede im Unternehmen wieder herzustellen, um die qualifizierten Kräfte nicht zu demotivieren. Je nach Arbeitsmarktlage und Ersetzbarkeit der Beschäftigten können aber auch negative ripple-Effekte auftreten, wie dies z.B. in Großbritannien sowie in Ostdeutschland der Fall war

Ganz offensichtlich hängen diese Effekte von der Gesamtarchitektur der Lohnsysteme ab. Wir haben insgesamt sechs unterschiedliche Typen identifiziert, die sich hinsichtlich des Verhältnisses und der Interaktion zwischen Mindestlöhnen und tariflicher Regelungen sowie deren Wirkungen auf die Lohnungleichheit unterscheiden. Im Typus 
des isolierten Mindestlohnes (Beispiel Vereinigtes Königreich) ohne die Kombination mit inklusiven Tarifverträgen hängen die ripple-Effekte von Unternehmensentscheidungen ab und sind je nach Qualifikationsstruktur der Beschäftigten und Arbeitsmarktlage variabel. Im Typus des extensiven Mindestlohnes (Ungarn) versucht der Staat, aufgrund fehlender autonomer Lohnverhandlungen durch nach Qualifikation gestaffelte Mindestlöhne Lohndifferenzen auf dem Arbeitsmarkt zu etablieren. Im Typus der direkten Interaktion (Frankreich) konzentrieren sich Politik und Gewerkschaften auf die Erhöhung des Mindestlohnes, die sich über allgemeinverbindliche Tarifverträge auch in das gesamte Lohngefüge übersetzt. Beim Typus der distanzierten Koexistenz (Belgien, Niederlande, Spanien) ist die Koppelung nicht so eng, da in vielen Branchen die Tariflöhne über den Mindestlöhnen liegen. Im Typus der autonomen Tarifpolitik (Dänemark, Österreich, Schweden) gibt es keinen gesetzlichen Mindestlohn. Die Tarifpartner setzen durch die hohe Tarifbindung jedoch wirkungsvolle Lohnuntergrenzen und benötigen hierzu aufgrund ihrer Organisationsstärke keine staatliche Hilfe. Deutschland wurde als Hybridmodell eingestuft, das mangels eines dominanten Modells Merkmale unterschiedlicher Modelle aufweist.

Die neuere Forschung zu den Branchenmindestlöhnen in Deutschland hat gezeigt, dass diese in Westdeutschland in den meisten Branchen die gesamte Lohnstruktur angehoben haben, während sie in Ostdeutschland komprimiert worden ist. Der Versuch, dies über höhere Mindestlöhne für Fachkräfte in einzelnen Branchen zu verhindern, war in Ostdeutschland nicht erfolgreich, weil deren Einhaltung selbst über staatliche Kontrollen nicht garantiert werden konnte. Dies verweist darauf, dass sich künftige Forschung nicht alleine auf die Untersuchung formeller Lohnstrukturen konzentrieren kann, sondern viel stärker auch ihre wirksame Durchsetzung in der Praxis in den Blick nehmen muss.

Die hier entwickelte Typologie ist (wie alle Typologien) statisch. Für die weitere Forschung ist es zentral, auch die Entwicklung der Institutionen zur Lohnfindung genauer zu untersuchen. Wir wissen sehr viel darüber, wie inklusive Lohnsysteme geschwächt werden können - etwa durch Abschaffung der Allgemeinverbindlichkeit von Tarifverträgen, die Auflösung von Branchenorganisationen der Unternehmer oder ihre Weigerung, weiterhin Branchentarifverträge auszuhandeln, oder auch durch die Einschränkung gewerkschaftlicher Streikaktionen. Viel zu wenig wissen wir aber über Ansatzpunkte zur Stärkung oder Wiederherstellung inklusiver Lohnsysteme.

\section{LITERATUR}

Banyuls, J./Cano, E./Aguado, E. (2010): National Report Spain. EC project Minimum Wage Systems and Changing Industrial Relations in Europe, Manchester, http://research.mbs.ac.uk/european-employment/Portals/0/docs/spanishreport.pdf

Bezzina, E. (2012): In 2010, 17\% of employees in the EU were low-wage earners: Eurostat, Statistics in focus 48/2012

Bispinck, R. (2012): Allgemeinverbindlicherklärung von Tarifverträgen - vom Niedergang zu Reform?, in: WSI-Mitteilungen 65 (7), S. 496-507, http://www. boeckler.de/wsimit_2012_07_bispinck.pdf

Blau, F. D./Kahn, L. M. (1994): International Differences in Male Wage Inequality: Institutions versus Market Forces, Working Paper (4678), Cambridge Bosch, G. (2013): Grenzüberschreitende Arbeitsmärkte und nationale Beschäftigungssysteme in der EU, in: Soeffner, H.-G. (Hrsg.): Transnationale Vergesellschaftungen, Verhandlungen des 35. Kongresses der Deutschen Gesellschaft für Soziologie in Frankfurt am Main 2010, Wiesbaden, S. 279-292
Bosch, G./Mayhew, K./Gautié, J. (2010): Industrial relations, legal regulations, and wage setting, in: Gautié, J./Schmitt, J. (Hrsg.): Low-Wage Work in the Wealthy World, New York, S. 147-182

Bosch, G./Weinkopf, C. (2012a): Wirkungen der Mindestlohnregelungen in acht Branchen, Expertise im Auftrag der Friedrich-Ebert-Stiftung (FES), Bonn Bosch, G./Weinkopf, C. (2012b): Mindestlöhne, Tariflöhne und Lohnungleichheit, in: Bispinck R./Bosch, G./Hofemann K./Nägele G. (Hrsg.): Sozialpolitik und Sozialstaat, Wiesbaden, S. 221-238

Bosch, G./Weinkopf, C./Worthmann, G. (2011): Die Fragilität des Tarifsystems: Einhaltung von Entgeltstandards und Mindestlöhnen am Beispiel des Bauhauptgewerbes, Berlin

Caroli, E./Gautié, J. (Hrsg.) (2008): Low-Wage Work in France, New York Dunlop, J. T. (1957): The Task of Contemporary Wage Theory, in: Taylor, G. W./ Pierson, F. C. (Hrsg.): New Concepts in Wage Determination, New York/Toronto/London, S. 117-139

Eldring, L./Alsos, K. (2012): European Minimum Wage: A Nordic Outlook: Fafo-report 16/2012, Oslo

Ellguth, P./Kohaut, S. (2012): Tarifbindung und betriebliche Interessenvertretung: Aktuelle Ergebnisse aus dem IAB-Betriebspanel 2011, in: WSI-Mitteilungen 65 (4), S. 297-305, http://www.boeckler.de/wsimit_2012_04_ellguth.pdf European Commission (2009): Industrial Relations 2008, Luxemburg Grimshaw, D. (2010): United Kingdom: Developing a progressive minimum wage in a liberal market economy, in: Vaughan-Whitehead, D. (Hrsg.): The Minimum Wage Revisited in the Enlarged EU, Cheltenham/Geneva, S. 473-508 Grimshaw, D. (2013) (Hrsg.): Minimum Wages, Pay Equity and Comparative Industrial Relations, London

Grimshaw, D./Bosch, G. (2013): The intersection between Minimum Wage and Collective Bargaining Institutions, in: Grimshaw, D. (Hrsg.), a. a. O., S. 51-81 Grimshaw, D./Bosch, G./Rubery, J. (2013): Minimum Wages and Egalitarian Pay Bargaining in Comparative Perspective, in: Grimshaw, D. (Hrsg.), a. a. O., S. $227-260$

Grimshaw, D./Rubery, J. (2013): The distributive functions of a minimum wage: first and second-order pay equity effects, in: Grimshaw, D. (Hrsg.), a. a. O., S. 82-114

Grimshaw, D./Shepherd, K./Rubery, J. (2010): National Report UK, EC project Minimum Wage Systems and Changing Industrial Relations in Europe, Manchester, http://research.mbs.ac.uk/european-employment/Portals/0/docs/ UKReport_000.pdf

Hayter, S./Weinberg, B. (2011): Mind the gap: collective bargaining and wage inequality, in: Hayter, S. (Hrsg.): The role of collective bargaining in the global economy. Negotiating for social justice, Cheltenham, S. 136-186

Kalina, T./Weinkopf, C. (2012): Niedriglohnbeschäftigung 2010: Fast jede/r Vierte arbeitet für Niedriglohn, IAQ-Report 2012-01, Duisburg

Koubi, M./Lhommeau, B. (2007): Les effets de diffusion de court terme des hausses du Smic dans les grilles salariales des enterprises de dix salariés ou plus sur la période 2000-2005, Les salaires en France, édition 2007

Low Pay Commission (LPC) (2007): National Minimum Wage. Low Pay Commission Report 2007. The Stationary Office, London, http://www.lowpay.gov. uk/lowpay/rep_a_p_index.shtml

Mason, G./Salverda, W. (2010): Low pay, working conditions and living standards, in: Gautié, J./Schmitt, J. (Hrsg.): Low-Wage Work in the Wealthy World, New York, S. 35-90

Ministère du Travail, de l'Emploi, de la Formation Professionelle et du Dialogue Social (2013): L'allègement de charges patronales sur les bas et moyens salaires, http://travail-emploi.gouv.fr/informations-pratiques,89/fichespratiques,91/remuneration,113/l-allegement-de-charges-patronales,1031.htm Neumann, L. (2010): National Report Hungary. EC project - Minimum Wage Systems and Changing Industrial Relations in Europe, Manchester, http://research.mbs.ac.uk/european-employment/Portals/0/docs/HungarianReport. pdf

Salverda, W. (2008): Labor Market Institutions, Low-Wage Work, and Job Qualiy, in: Salverda, W./van Klaveren, M./van der Meer, M. (Hrsg.): Low-Wage Work in the Netherlands, New York, S. 63-131

Salverda, W./Mayhew, K. (2009): Capitalist economies and wage inequality, in: Oxford Review of Economic Policy 25 (1), S. 126-154

Schulten, T. (2012a): WSI-Mindestlohnbericht 2012 - Schwache Mindestlohnentwicklung unter staatlicher Austeritätspolitik, in: WSI-Mitteilungen 65 (2), S. 124-130, http://www.boeckler.de/wsimit_2012_02_schulten.pdf

Schulten, T. (2012b): Stellenwert der Allgemeinverbindlicherklärung für die Tarifvertragssysteme in Europa, in: WSI-Mitteilungen 65 (7), S. 485-495, http:// www.boeckler.de/wsimit_2012_07_schulten.pdf 
Sniekers, F. (2011): The changing impact of labour market institutions on earnings inequality. A study of the effects of trade unions and the minimum wage in advanced industrial countries since the 1980s: Tinbergen Institut, Amsterdam

Stewart, M. B. (2010): Individual-level Wage Changes and Spillover Effects of Minimum Wage Increases: University of Warwick, ESRC

Visser, J. (2011): Institutional Characteristics of Trade Unions, Wage Setting,

State Intervention and Social Pacts (ICTWSS). An international database: Amsterdam Institute for Advanced Labour Studies (AIAS), Amsterdam, http:// www.uva-aias.net/208

Westergaard-Nielsen, N. (Hrsg.) (2008): Low-Wage Work in Denmark, New York

Wicks-Lim, J. (2008): Mandated wage floors and the wage structure: new estimates of the ripple effects of minimum wage laws, in: Pollin, R./Brenner, M./ Wicks-Lim, J./Luce, S. (Hrsg.): A measure of fairness the economics of living wages and minimum wages in the United States, Ithaca, S. 199-215

\section{AUTOREN}

GERHARD BOSCH, Prof. Dr., ist Geschäftsführender Direktor des Institut Arbeit und Qualifikation (IAQ) an der Universität Duisburg-Essen. Arbeitsschwerpunkte: Arbeitsmarkt- und Strukturpolitik, Arbeitszeit, Personalpolitik.

@gerhard.bosch@uni-due.de

CLAUDIA WEINKOPF, Dr., ist Stellvertretende Geschäftsführende Direktorin des IAQ und Leiterin der Forschungsabteilung Flexibilität und Sicherheit. Ar beitsschwerpunkte: Arbeitsmarkt und Beschäftigung, Personalpolitik, Niedrigund Mindestlöhne, Gender.

@ claudia.weinkopf@uni-due.de 